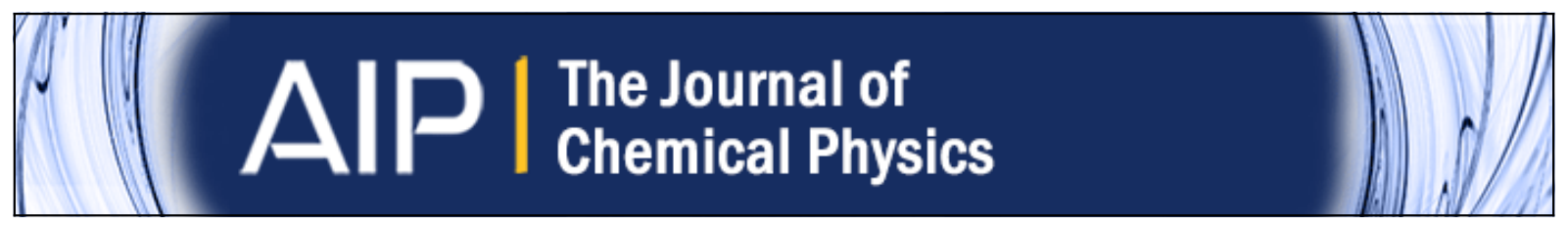

Quantum state specific reactant preparation in a molecular beam by rapid adiabatic passage

Helen Chadwick, P. Morten Hundt, Maarten E. van Reijzen, Bruce L. Yoder, and Rainer D. Beck

Citation: The Journal of Chemical Physics 140, 034321 (2014); doi: 10.1063/1.4861054

View online: http://dx.doi.org/10.1063/1.4861054

View Table of Contents: http://scitation.aip.org/content/aip/journal/jcp/140/3?ver=pdfcov

Published by the AIP Publishing

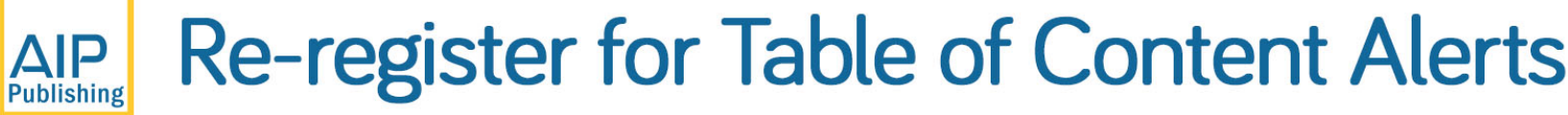

\section{Create a profile.




\title{
Quantum state specific reactant preparation in a molecular beam by rapid adiabatic passage
}

\author{
Helen Chadwick, ${ }^{\text {a) }}$ P. Morten Hundt, Maarten E. van Reijzen, Bruce L. Yoder, \\ and Rainer D. Beck \\ Laboratoire de Chimie Physique Moléculaire, Ecole Polytechnique Fédérale de Lausanne, \\ Lausanne, Switzerland
}

(Received 30 October 2013; accepted 18 December 2013; published online 21 January 2014)

\begin{abstract}
Highly efficient preparation of molecules in a specific rovibrationally excited state for gas/surface reactivity measurements is achieved in a molecular beam using tunable infrared (IR) radiation from a single mode continuous wave optical parametric oscillator (cw-OPO). We demonstrate that with appropriate focusing of the IR radiation, molecules in the molecular beam crossing the fixed frequency IR field experience a Doppler tuning that can be adjusted to achieve complete population inversion of a two-level system by rapid adiabatic passage (RAP). A room temperature pyroelectric detector is used to monitor the excited fraction in the molecular beam and the population inversion is detected and quantified using IR bleaching by a second IR-OPO. The second OPO is also used for complete population transfer to an overtone or combination vibration via double resonance excitation using two spatially separated RAP processes. () 2014 AIP Publishing LLC. [http://dx.doi.org/10.1063/1.4861054]
\end{abstract}

\section{INTRODUCTION}

A central goal of reaction dynamics is to gain fundamental insight into chemical processes at a microscopic level. This presents a complex problem, due to the number of translational, rotational, and vibrational degrees of freedom that can be associated with the interaction. Being able to exert control on the initial state of the reactants can, therefore, help to unravel the significance each degree of freedom has on the underlying dynamics. Detailed quantum state resolved reactivity studies of collisions at the gassurface interface have illustrated the additional information that can be obtained through such initial state preparation, by revealing the influence of the ro-vibrational state of the molecule on the outcome of the collision. ${ }^{1-4}$ For example, it has been shown that the chemisorption of methane on transition metal surfaces is both bond ${ }^{5,6}$ and mode ${ }^{7,8}$ specific, as well as being dependent on the alignment of the impinging molecule. ${ }^{9}$

In order to realize such quantum state resolved reactivity measurements, a significant fraction of the reactant molecules needs to be prepared in a specific ro-vibrational eigenstate. Theoretically, it is possible to produce a population inversion by tuning the laser power and interaction time to create an optical $\pi$-pulse, ${ }^{10,11}$ such that the molecules undergo half of a Rabi cycle, analogous to a radio frequency $\pi$-pulse used to invert a population of nuclear spins in NMR. However, this technique cannot be applied practically for optical excitation of molecules since the distribution of molecular speeds and variations in laser intensity present under typical experimental conditions will make it nearly impossible to create an optical $\pi$ pulse for a finite sample volume using a realistic laser

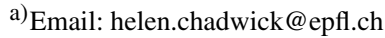

beam. Furthermore, transitions between degenerate $m$ levels within a given $J$ level will have different Rabi frequencies due to the different transition strengths. Therefore the excitation probability will be averaged over many different Rabi frequencies, with the result that at best half the population will be excited, a result which could also be achieved with incoherent excitation.

A more robust method for creating a population inversion is through rapid adiabatic passage (RAP), ${ }^{10,12-17}$ as will be considered here. This technique has previously been described theoretically ${ }^{10,12,18}$ and demonstrated experimentally in atomic systems including metastable helium ${ }^{17}$ and dysprosium. ${ }^{16}$ RAP has also been demonstrated for molecules such as $\mathrm{SF}_{6}{ }^{19}$ and $\mathrm{C}_{2} \mathrm{H}_{4}{ }^{12}$ where there are coincidental resonances with specific transitions of line tunable $\mathrm{CO}_{2}$ lasers. The advent of new widely tunable, powerful, single mode mid infrared (IR) optical parametric oscillators (OPOs) has opened up the possibility of exciting a large range of rovibrational transitions of many molecules, making RAP a valuable tool for quantum state specific reactant preparation, which we have recently implemented for quantum state resolved gas/surface reactivity measurements for $\mathrm{CH}_{4}{ }^{9,20,21}$ and $\mathrm{D}_{2} \mathrm{O} .{ }^{22}$

The remainder of the paper is organized as follows. In Sec. II, a theoretical description of RAP is presented and the conditions for creating a population inversion are introduced. After, a two-laser bleaching experiment is described which is used to quantify the population inversion produced by RAP excitation in a molecular beam. We also show that two sequential RAP steps can be used in a double resonance scheme for complete population transfer from the vibrational ground state $(v=0)$ to an overtone state $(v=2)$ of methane. The results of numerical simulations for the IR laser pumping in a molecular beam are then presented and compared to data 


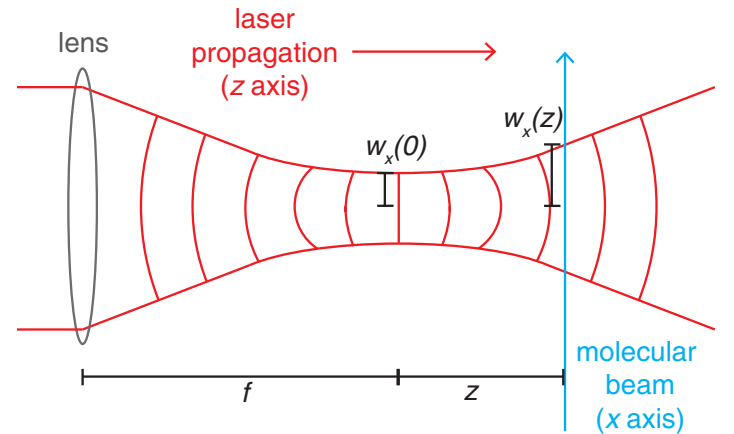

FIG. 1. A schematic representation of the focused laser beam, showing the focal length $(f)$, the laser beam waist at $z=0 \mathrm{~cm}\left(w_{x}(0)\right)$, and the laser beam waist at the crossing point of the molecular beam $\left(w_{x}(z)\right)$.

obtained experimentally. The final section summarizes the key points.

\section{RAPID ADIABATIC PASSAGE}

Excitation via $\mathrm{RAP}^{10,12-15}$ can be used to create a population inversion in a two level system through the use of coherent laser excitation. By sweeping the frequency of the excitation field across the molecular resonance it is possible to achieve a level crossing for a two level system in the presence of a resonant electromagnetic field. ${ }^{12}$ If the frequency sweep is sufficiently slow, adiabatic following occurs resulting in complete population transfer from the ground to the excited state. ${ }^{10-12}$ The creation of this population inversion is relatively insensitive to precise experimental conditions including the velocity of the molecules, changes in laser intensity and the different transition strengths corresponding to degenerate $m$ sublevels in a given $J$ level, making this a widely applicable method for obtaining complete population transfer.

For experiments that prepare reactant molecules in a molecular beam Doppler tuning provides a convenient way to create the required frequency sweep necessary for RAP. This can be achieved by focusing a fixed frequency continu- ous wave laser beam using a lens to create curved wavefronts, as shown schematically in Figure 1. For a Gaussian beam the waist at the focal point $(z=0 \mathrm{~cm})$ can be calculated $v_{i a}{ }^{23}$

$$
w_{x}(0)=\frac{f \lambda}{r \pi} .
$$

$f$ is the focal length of the lens, $r$ is the radius of the Gaussian beam at the position of the lens (corresponding to 1/e of the maximum laser power), and $\lambda$ is the wavelength of the light. The beam waist of the laser at a distance $z$ from the focal point can be found using ${ }^{24}$

$$
w_{x}(z)=w_{x}(0) \sqrt{1+\frac{z^{2}}{z_{R}^{2}}},
$$

where $z_{R}$ is the Rayleigh range, which is given by

$$
z_{R}=\frac{\pi w_{x}(0)^{2}}{\lambda} .
$$

The radius of curvature of the wavefronts at position $z$ can then be found $v i a^{24}$

$$
R(z)=z+\frac{z_{R}^{2}}{z}
$$

The dependence of the radius of curvature and the laser beam waist on the distance from the focal point is presented in the left and right panels of Figure 2, respectively, for a focal length of $f=10 \mathrm{~cm}$ (red), $f=25.4 \mathrm{~cm}$ (black), $f=40$ $\mathrm{cm}$ (green), $f=65 \mathrm{~cm}$ (blue), and $f=96 \mathrm{~cm}$ (purple). For the calculations, values of $\lambda=3.30 \mu \mathrm{m}$ and $r=2 \mathrm{~mm}$ were used.

As the molecular beam crosses the laser beam at a distance $z$ from the focal point, the molecules in the beam experience a frequency sweep due to the Doppler effect. The rate of this frequency sweep due to the curved wavefronts can be found using ${ }^{25}$

$$
\frac{d \phi}{d t}=\frac{v_{x}^{2} \omega_{\mathrm{B}}}{R(z) c},
$$

where $v_{x}$ is the velocity of the molecule perpendicular to the laser propagation axis, $\omega_{\mathrm{B}}$ the transition frequency and $c$ the
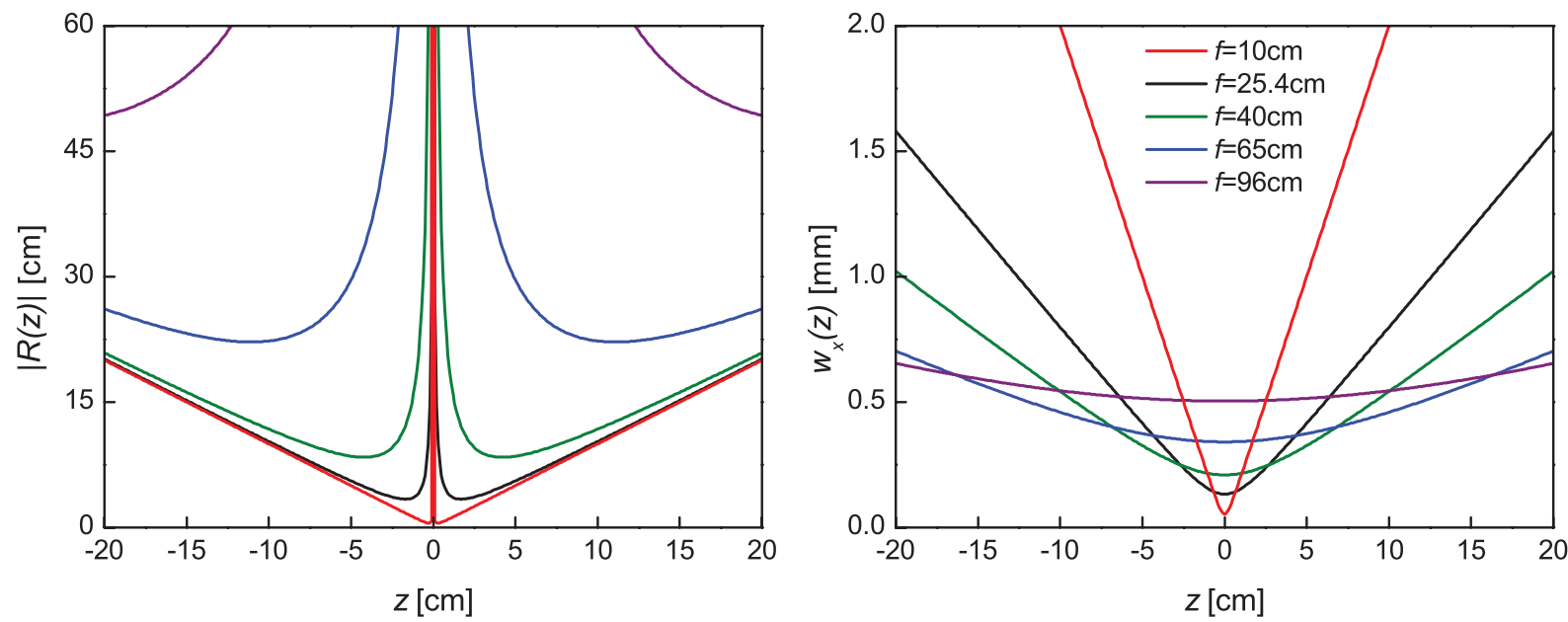

FIG. 2. The radius of wavefront curvature $R(z)$ (left) and Gaussian $1 / \mathrm{e}$ laser beam diameter $w_{x}(z)$ (right) as a function of the distance from the focused waist of the laser for a lens with focal length $f=10 \mathrm{~cm}$ (red), $f=25.4 \mathrm{~cm}$ (black), $f=40 \mathrm{~cm}$ (green), $f=65 \mathrm{~cm}$ (blue), and $f=96 \mathrm{~cm}$ (purple). 

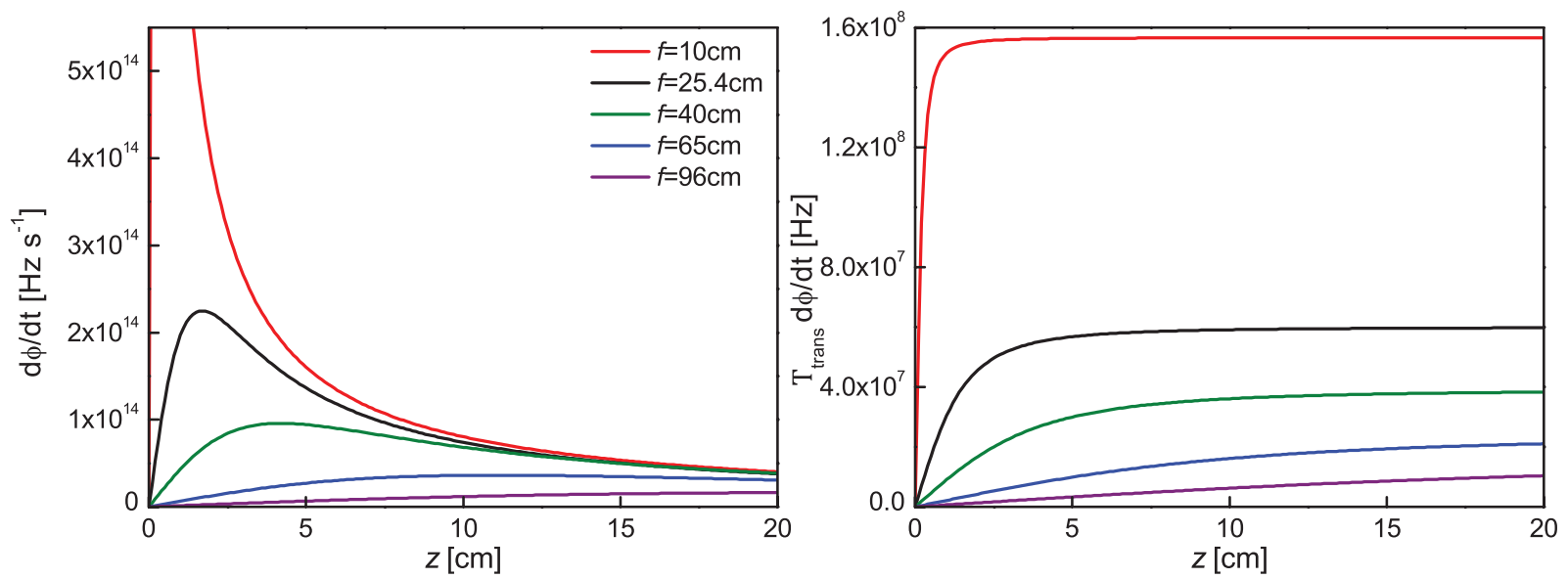

FIG. 3. The frequency sweep rate (left) and total sweep (right) experienced by a $\mathrm{CH}_{4}$ molecule crossing the IR beam with a velocity of $2056 \mathrm{~m} \mathrm{~s}{ }^{-1}$ at a distance $z$ from the focused waist of the laser beam for a lens of focal length $f=10 \mathrm{~cm}$ (red), $f=25.4 \mathrm{~cm}$ (black), $f=40 \mathrm{~cm}$ (green), $f=65 \mathrm{~cm}$ (blue), and $f=96 \mathrm{~cm}$ (purple).

speed of light. The total sweep experienced by the molecule from the curved wavefronts can be calculated via

$$
\frac{d \phi}{d t} T_{\text {trans }}=\frac{v_{x}^{2} \omega_{\mathrm{B}}}{R(z) c} T_{\text {trans }}
$$

where $T_{\text {trans }}$ is the time taken for the molecule to cross the laser beam, which is simply given by

$$
T_{\text {trans }}=\frac{2 w_{x}(z)}{v_{x}} .
$$

The sweep rate (left) and total sweep (right) due to the curved wavefronts at a distance $z$ from the focal point of the laser are shown in Figure 3 for a molecular beam travelling at $2056 \mathrm{~ms}^{-1}$, using a lens with focal length $f=10 \mathrm{~cm}$ (red), $f=25.4 \mathrm{~cm}$ (black), $f=40 \mathrm{~cm}$ (green), $f=65 \mathrm{~cm}$ (blue), and $f=96 \mathrm{~cm}$ (purple). As the distance between the focal point of the laser and molecular beam increases, the sweep rate decreases due to the increasing radius of curvature. However, the total sweep remains the same, reflecting the increasing time taken for the molecule to cross the diverging laser beam. As the focal length of the lens gets larger, both the sweep rate and sweep due to the curved wavefronts decreases, again due to the larger radius of curvature.

The total frequency sweep $(\Delta(t))$ experienced by a molecule at a time $t$ can be calculated as ${ }^{25}$

$$
\Delta(t)=\Delta \omega-\omega_{\mathrm{L}}-\frac{d \phi}{d t} t,
$$

where $\Delta \omega$ is the frequency difference between the initial and final level, $\omega_{\mathrm{L}}$ the laser frequency and $t=0$ corresponds to the molecule being at the center of the laser beam. It follows that if the laser is resonant with the transition and for a non-divergent molecular beam, the sweep is only due to the curved wavefronts. However, any angular divergence of the molecular beam will result in a time independent term due to the Doppler shift associated with the molecules velocity component parallel to the laser propagation direction (along $v_{z}$ ). Therefore the total sweep at time $t$ is

$$
\Delta(t)=\frac{v_{z} \omega_{\mathrm{B}}}{c}-\frac{v_{x}^{2} \omega_{\mathrm{B}}}{R(z) c} t .
$$

To achieve RAP, it is also necessary to consider the strength of the interaction of the molecule with the electric field of the laser. This is characterized by the Rabi frequency, given by ${ }^{25}$

$$
\Omega(z)=\frac{\mu \cdot E}{\hbar} .
$$

The transition dipole moment, $\mu$, is related to the Einstein coefficients $A_{21}$ via ${ }^{25}$

$$
\mu=\left\langle J_{1} m_{1}, 10 \mid J_{2} m_{2}\right\rangle \sqrt{\frac{3 \varepsilon_{0} h c^{3}}{2 \omega_{\mathrm{B}}^{3}} A_{21}} .
$$

$\left\langle J_{1} m_{1}, 10 \mid J_{2} m_{2}\right\rangle$ is a Clebsch-Gordan coefficient ${ }^{26}$ that accounts for the dependence of the transition probability on the rotational quantum numbers.

Assuming a Gaussian beam profile for the laser, the electric field, $E$ is given by

$$
E=E_{0} \exp \left(-\frac{x^{2}}{w_{x}(z)^{2}}-\frac{y^{2}}{w_{y}(z)^{2}}\right)
$$

where $E_{0}$ is the peak intensity which can be calculated via

$$
E_{0}=\sqrt{\frac{2 I}{\varepsilon_{0} c}},
$$

where $\varepsilon_{0}$ is the vacuum permittivity $\left(8.85 \times 10^{-12} \mathrm{~F} \mathrm{~m}^{-1}\right)$. The laser intensity, $I$, is given by

$$
I=\frac{2 P}{A},
$$

where $A$ is the area of the laser beam $\left(A=\pi w_{x}(z) w_{y}(z)\right)$.

The dependence of the Rabi frequency on $z$ is presented in Figure 4 for a laser power of $0.5 \mathrm{~W}$. The calculations were performed using a value of $\mu=1.8 \times 10^{-31} \mathrm{C} \mathrm{m},{ }^{27}$ corresponding to excitation of the $v_{3}$ vibration via the $\mathrm{R}(0)$ transition in $\mathrm{CH}_{4}$, for lenses with a focal length of $f=10 \mathrm{~cm}$ (red), $f=25.4 \mathrm{~cm}$ (black), $f=40 \mathrm{~cm}$ (blue), $f=65 \mathrm{~cm}$ (green), and $f=96 \mathrm{~cm}$ (purple). The decrease in Rabi frequency with increasing $z$ reflects the decreasing magnitude of $E$ as the laser beam diverges. The variation is most marked for the shortest focal length lens, while for the longest focal length lens 


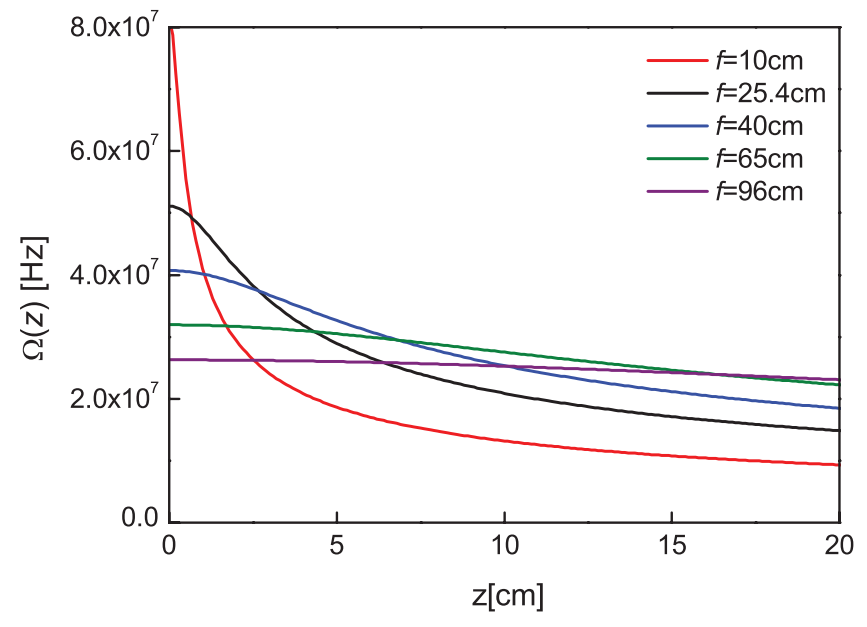

FIG. 4. The dependence of the Rabi frequency on the position from the laser beam focus for a lens with length $f=10 \mathrm{~cm}$ (red), $f=25.4 \mathrm{~cm}$ (black), $f=40$ $\mathrm{cm}$ (blue), $f=65 \mathrm{~cm}$ (green), and $f=96 \mathrm{~cm}$ (purple), calculated for a laser power of $0.5 \mathrm{~W}$.

the change in Rabi frequency is not as significant. Consequently, at a distance of $20 \mathrm{~cm}$ away from the focal point, the $f=96 \mathrm{~cm}$ lens produces the largest Rabi frequency, and the $f=10 \mathrm{~cm}$ lens the smallest.

To achieve RAP experimentally, a number of conditions have to be met. ${ }^{12,14}$ First, the frequency sweep rate has to be sufficiently slow to ensure an adiabatic following to transfer the population from the ground state to the excited state. At the same time, the total sweep amplitude given by the product of the sweep rate and transit time must be larger than the Rabi frequency to ensure that the molecules experience a frequency sweep while passing through the laser beam that starts and ends out of resonance. The variation in laser power must also be smooth, i.e., there should be no sudden switching in the intensity of the electric field. If these three conditions are met, it is possible to calculate the excitation probability using the Landau-Zener formula, given by ${ }^{10}$

$$
\text { Probability }=1-\exp \left(-\frac{\pi \Omega(z)^{2}}{2\left|\frac{d \phi}{d t}\right|}\right) .
$$

A more general method for simulating the RAP process for a two level system is to solve the optical Bloch equations, as will be discussed in Sec. IV.

\section{EXPERIMENT}

The experimental methods used have been described in detail previously, ${ }^{21,23,28}$ and so only a brief summary will be presented here. Reactant excitation in a specific ro-vibrational eigenstate is performed using a tunable, single mode, continuous wave IR optical parametric oscillator (Argos Model 2400SF, Module C, Lockheed Martin Acculight Corp.). The OPO idler output has an intrinsic linewidth of less than $1 \mathrm{MHz}$ on the timescale of seconds and is stabilized to $\pm 1 \mathrm{MHz}$ on the timescale of hours by locking to a Lamb dip detected in a static gas cell filled with typically $10 \mathrm{~Pa}$ of the same molecule that is used in the molecular beam. The IR beam is focused by a cylindrical $\mathrm{CaF}_{2}$ lens with a focal length of $f=25.4 \mathrm{~cm}$ to create curved wavefronts resulting in a frequency sweep due to Doppler tuning for molecules crossing the molecular beam (see Figure 1). As a cylindrical lens is used, the laser is only focused in the $x$ direction along the direction of the molecular beam propagation and therefore $w_{y}(0)=w_{y}(z)=r$. The distance between the lens and molecular beam was set to $36 \mathrm{~cm}$, corresponding to a value of $z=10.6 \mathrm{~cm}$. To monitor the fraction of vibrationally excited molecules prepared by IR-pumping, a room temperature pyroelectric detector is inserted into the molecular beam after the laser crossing region to monitor its vibrational energy content. Vibrationally excited molecules in the beam are detected selectively using lock-in detection in combination with chopping of the excitation laser beam at $2 \mathrm{~Hz}$ using an electromagnetic shutter.

The two laser bleaching experiment performed to confirm and quantify the population inversion created by RAP for single photon excitation is shown schematically in the top center panel of Figure 5. Initially only OPO-1 is used to excite the $\mathrm{CH}_{4}\left(v_{3}\right)$ vibration via the $\mathrm{P}(1)$ transition $\left(\mu=1.04 \times 10^{-31}\right.$ $\mathrm{Cm}^{27}$ ), and the resulting pyroelectric detector signal recorded via phase sensitive detection tuned to the chopping frequency of OPO-1, as shown in the top right hand panel of the figure. When the second unchopped OPO beam is added upstream to excite molecules via the $\mathrm{R}(1)$ transition, the pyroelectric signal almost disappears since the detector only responds to molecules excited by amplitude modulated radiation from OPO-1. As both OPOs are tuned to transitions originating from the same $J=1$ initial state, this shows that OPO-2 effectively removes most of the population from that state leaving almost no molecules for OPO-1 to excite, and therefore demonstrates the creation of the population inversion. The small remaining signal $(<5 \%$ of the signal with OPO-1 only) observed when both OPOs are on resonance most likely reflects imperfect spatial overlap of the molecular beam volumes excited by the two OPOs. Finally, the pyroelectric detector signal level due to scattered IR radiation is recorded with both OPOs intersecting the molecular beam but off resonance to establish a baseline for the signal due to laser excited molecules.

The bottom row of Figure 5 shows a similar experiment that was done to verify that it is also possible to achieve complete population transfer from the initial $v=0$ state of $\mathrm{CH}_{4}$ to a $2 v_{3}$ overtone state through two sequential RAP processes using double resonance excitation. As with the bleaching experiment described above, OPO- 1 is chopped at $2 \mathrm{~Hz}$ and tuned to excite the $\mathrm{CH}_{4}\left(v_{3}\right)$ vibration via the $\mathrm{P}(1)$ transition, and the resulting signal monitored with the pyroelectric detector. This is shown in the bottom right panel. However, OPO-2 is now tuned to transfer the population from $v_{3}$ to $2 v_{3}$ via the $\mathrm{R}(0)$ transition of the E symmetry. ${ }^{29}$ When both OPOs are used simultaneously to excite the molecular beam, the pyroelectric detector signal approximately doubles compared to the signal obtained with OPO-1 only even though OPO-2 is not chopped. Assuming that the efficiency of the molecules transferring their vibrational energy to the pyroelectric detector is independent of the vibrational state, the doubling of the pyroelectric detector signal confirms that all of the molecules excited by OPO- 1 to $v_{3}$ are further excited to 

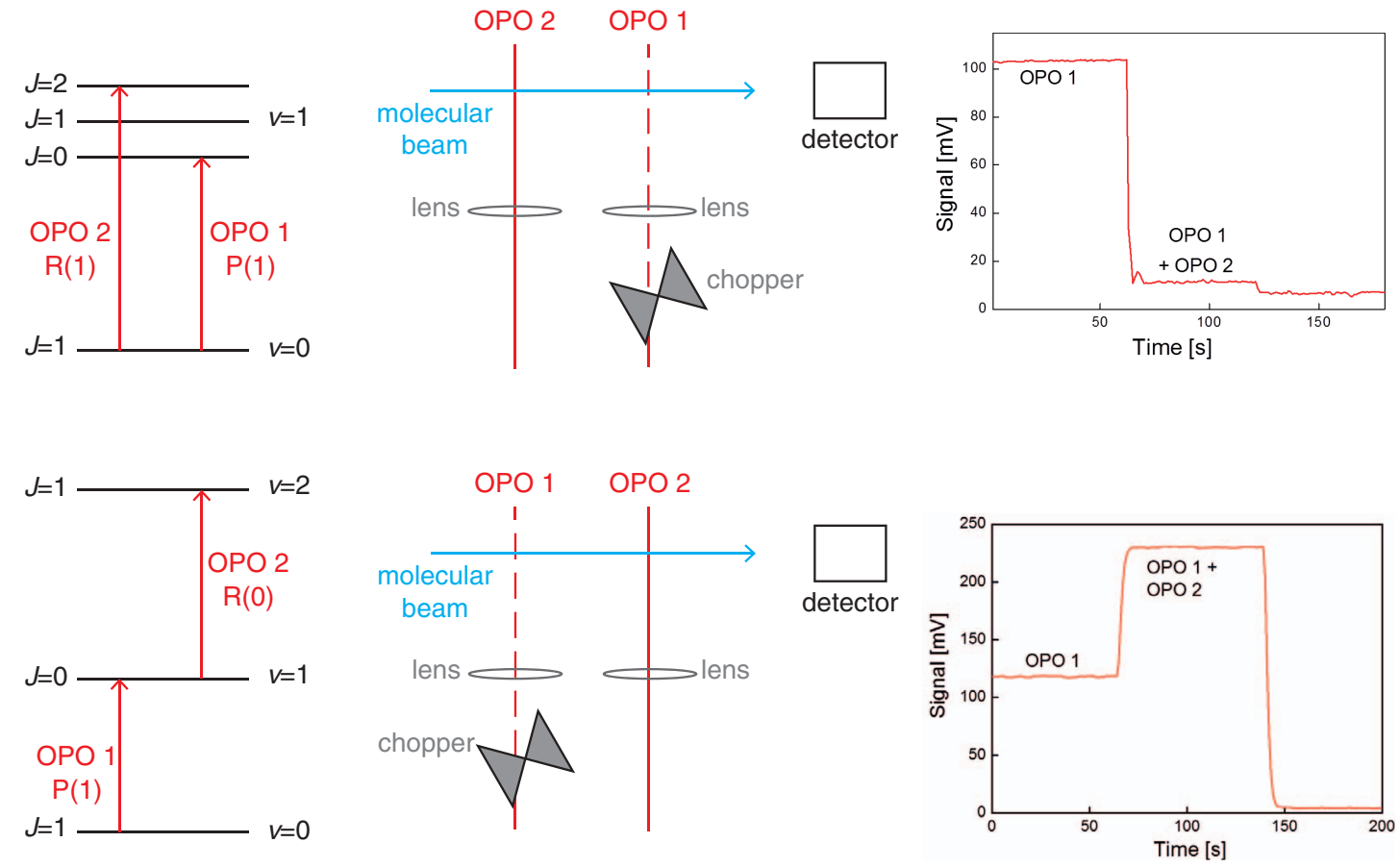

FIG. 5. A schematic representation of the method used to detect the population inversion produced by the RAP excitation process (middle), energy level diagram and transitions used for excitation (left), and the resulting detector signal (right) for single resonance (top) and double resonance (bottom) excitation.

$2 v_{3}$ by OPO-2, and therefore all the population of the ground state has been successfully transferred through two sequential RAP processes.

The excitation scheme described above can be used for reactant preparation in an overtone or combination level containing two quanta of vibrational excitation corresponding to $\mathrm{h} \nu_{\mathrm{OPO}-1}$ and $\mathrm{h} \nu_{\mathrm{OPO}-2}$. Double resonance excitation also makes it possible to prepare the molecule in a low frequency vibration $v=v_{\mathrm{OPO}-1}-v_{\mathrm{OPO}-2}$ which can lie outside the tuning range of either of the OPO sources (e.g., $v_{4}$ the infrared active bending fundamental of $\mathrm{CH}_{4}$ ) or to excite a Raman active vibration that is inaccessible from ground state by a single photon transition (e.g., $v_{2}$ of $\mathrm{CH}_{4}$ ). The selection rules for IR excitation also restrict the symmetry of the states that can be prepared using single photon excitation. For example, direct overtone pumping (i.e., a single photon transition) from the symmetric $\left(\mathrm{A}_{1}\right)$ ground state of $\mathrm{CH}_{4}$ would only allow $2 v_{3}$ to be prepared in the $\mathrm{F}_{2}$ state. However, it is possible to prepare the $A_{1}, F_{2}$ and $E$ symmetry states by using double resonance excitation, ${ }^{29-31}$ allowing a wider range of vibrational states to be studied. Using direct overtone pumping also results in a smaller fraction of the molecular beam being excited as the transition dipole moment is significantly smaller $(\mu=1.0$ $\left.\times 10^{-32} \mathrm{Cm}^{27}\right)$. The results from the calculations discussed in the following section estimate that approximately $2 \%$ of the molecules would be excited at a laser power of $1 \mathrm{~W}$, and that $40 \mathrm{~W}$ is required to excite half the molecules in direct overtone excitation.

Whilst RAP provides a robust method for creating a population inversion when the conditions outlined in Sec. II can be met, there are situations in which the technique will not result in complete population transfer. ${ }^{11}$ The slow sweep rate required to ensure that adiabatic following occurs requires a long interaction time of the molecule with the laser beam. If the excited state is short lived, the excited molecules can undergo spontaneous emission in this time and the population inversion will not be complete. This is also a potential problem for the double resonance excitation via sequential RAP processes described above, where it would be possible to lose population from the state prepared by the first laser through spontaneous emission or collisions, depending on the molecule and the conditions employed. There are alternative methods to create a population inversion in three level systems, for example, stimulated Raman adiabatic passage..$^{10,11,32,33}$ The advantage of this technique is that the intermediate state is never populated and therefore the properties of that level do not affect the probability the population is transferred between the initial and final state. It should be noted that these techniques can also be extended to multi-level systems. $^{10,12,33}$

\section{SIMULATIONS}

A method for simulating excitation through RAP for a two level system is to solve the optical Bloch equations, given by ${ }^{18,34-36}$

$$
\begin{gathered}
\frac{d U}{d t}=-\Delta V, \\
\frac{d V}{d t}=\Delta U+\Omega W, \\
\frac{d W}{d t}=-\Omega V,
\end{gathered}
$$



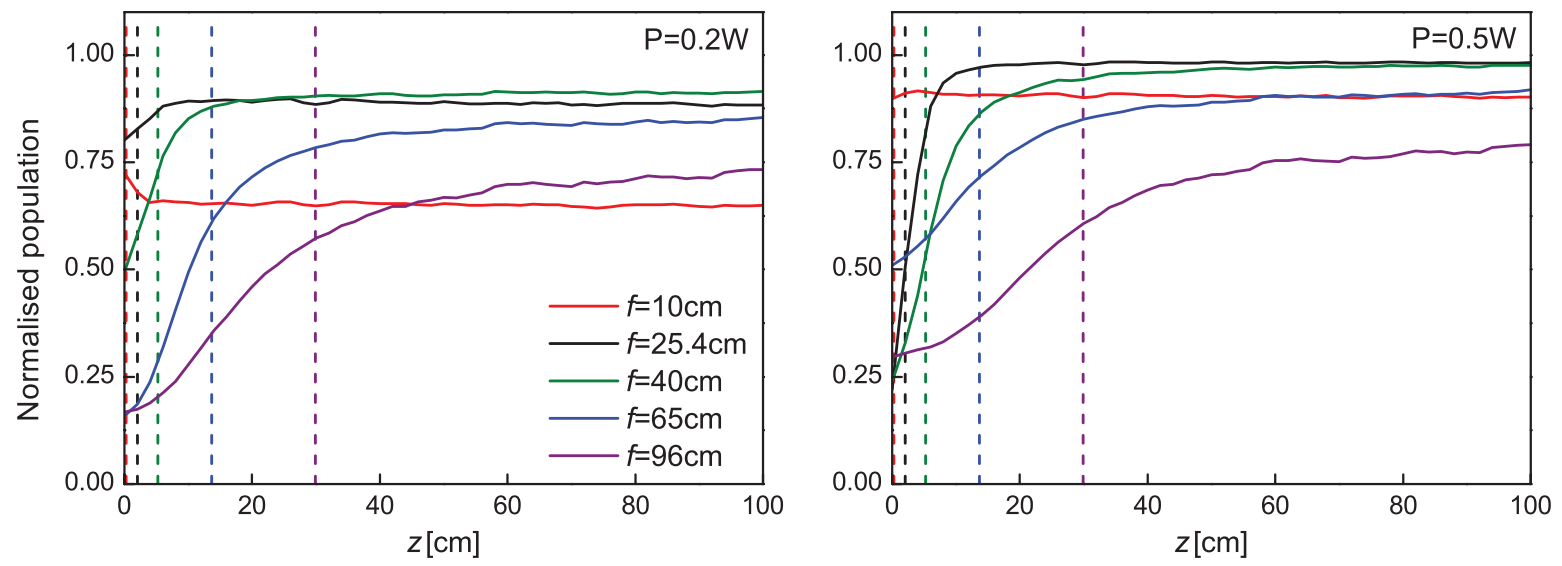

FIG. 6. The calculated $\nu_{3}$ excitation probability for $\mathrm{CH}_{4}$ crossing the IR laser beam at a distance $z$ from the focused laser beam waist created with a lens with a focal length of $10 \mathrm{~cm}$ (red), $25.4 \mathrm{~cm}$ (black), $40 \mathrm{~cm}$ (green), $65 \mathrm{~cm}$ (blue), and $96 \mathrm{~cm}$ (purple). The Rayleigh range is shown as a dashed line in the same color for each focal length. The left hand panel corresponds to a laser power of $0.2 \mathrm{~W}$ and the right hand panel corresponds to a laser power of $0.5 \mathrm{~W}$.

where $U$ and $-V$ are the components of the molecular dipole moment in phase and in quadrature with the laser electric field, ${ }^{34}$ and $\Delta$ and $\Omega$ are the frequency sweep and Rabi frequency as discussed in Sec. II. The excitation probability after passing through the laser can be found $v_{i a}{ }^{34}$

$$
\text { Probability }=\frac{1+W}{2} \text {. }
$$

The initial condition for the integration is that $V=0$, $U=0$, and $W=-1$, i.e., all the population is in the ground state before excitation. The equations were solved using a Runge-Kutta method ${ }^{37}$ and the interval of the integration was $3 T_{\text {trans. }}$.

Monte-Carlo simulations were performed for the excitation of the $v_{3}$ vibration of $\mathrm{CH}_{4}$. A molecular beam velocity of $2056 \mathrm{~ms}^{-1}$ was used as determined experimentally from time of flight measurements. ${ }^{9}$ The distribution of velocities in the molecular beam was modeled as a Gaussian with a full width at half maximum of $200 \mathrm{~ms}^{-1}$, with a full angular divergence of $0.7^{\circ}$ (resulting in a residual Doppler width (FWHM) of $\pm 3.8 \mathrm{MHz}$ ) for a transition frequency of $3000 \mathrm{~cm}^{-1}$. The

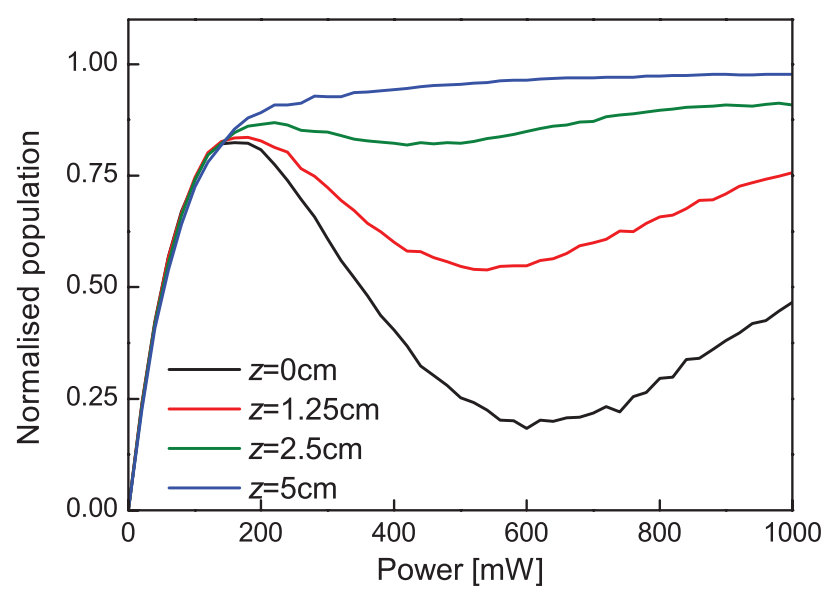

FIG. 7. Simulated power dependence for excitation of $\mathrm{CH}_{4}\left(\nu_{3}\right)$ for an IR beam focused by a $f=25.4 \mathrm{~cm}$ lens. The molecular beam crosses the IR laser at different positions $z$ : at the focus point $z=0 \mathrm{~cm}$ (black), $1.25 \mathrm{~cm}$ (red), $2.5 \mathrm{~cm}$ (green), and $5 \mathrm{~cm}$ (blue) away from the focus. radius of the unfocused laser beam was taken to be $2 \mathrm{~mm}$, which is in good agreement with the value of $1.9 \mathrm{~mm}$ measured experimentally. ${ }^{21}$

Simulations were performed to determine the probability of $v_{3}$ excitation via the $\mathrm{R}(0)$ transition for $\mathrm{CH}_{4}$ molecules after passing through the IR laser beam at different $z$ positions using a $10 \mathrm{~cm}$ (red), $25.4 \mathrm{~cm}$ (black), $40 \mathrm{~cm}$ (green), $65 \mathrm{~cm}$ (blue), and $96 \mathrm{~cm}$ (purple) focal length lens for a laser power of $0.2 \mathrm{~W}$ (left) and $0.5 \mathrm{~W}$ (right). The results are presented in Figure 6. The vertical dashed lines in the panels correspond to the Rayleigh range $z_{R}$ for each lens. When the molecular beam traverses the IR beam at $z=z_{R}$, the excitation probability is not maximum but increases further with increasing $z$ until it reaches an asymptote for $z \gg z_{R}$. Although the maximum sweep rate is at $z_{R}$, this position is not optimal for RAP as the laser beam waist is still small, and consequently the total sweep will be lower due to the shorter transit time and the Rabi frequency large due to the high electric field strength. Using the shortest focal length lens also results in the largest sweep rate, as shown in Figure 3. However here this sweep rate is too high compared to the Rabi frequency to allow adiabatic following to occur. As the focal length is increased, the excitation probability increases before decreasing for the longest focal lengths. This is due to the smaller total sweep produced by these lenses, which is then insufficient to excite the most divergent molecules in the molecular beam. However, these calculations show that with an appropriate choice of focal length and lens position it is possible to excite the full residual Doppler width of $7.6 \mathrm{MHz}( \pm 3.8 \mathrm{MHz})$ of the molecular beam. With the $25.4 \mathrm{~cm}$ focal length lens, it would be possible to excite molecules with an angular divergence of up to $0.9^{\circ}$ travelling at the velocity of $2056 \mathrm{~m} \mathrm{~s}^{-1}$ considered here.

The results from simulations for the same transition using a $25.4 \mathrm{~cm}$ focal length lens with the molecular beam crossing the laser beam at the focused waist (black), and at $1.25 \mathrm{~cm}$ (red), $2.5 \mathrm{~cm}$ (green), and $5 \mathrm{~cm}$ (blue) away from the focal point are presented in Figure 7. At $z=0 \mathrm{~cm}$ the molecular beam does not experience a frequency sweep so the laser is resonant with the transition between the ground and excited state throughout the whole transit time of the molecule. 

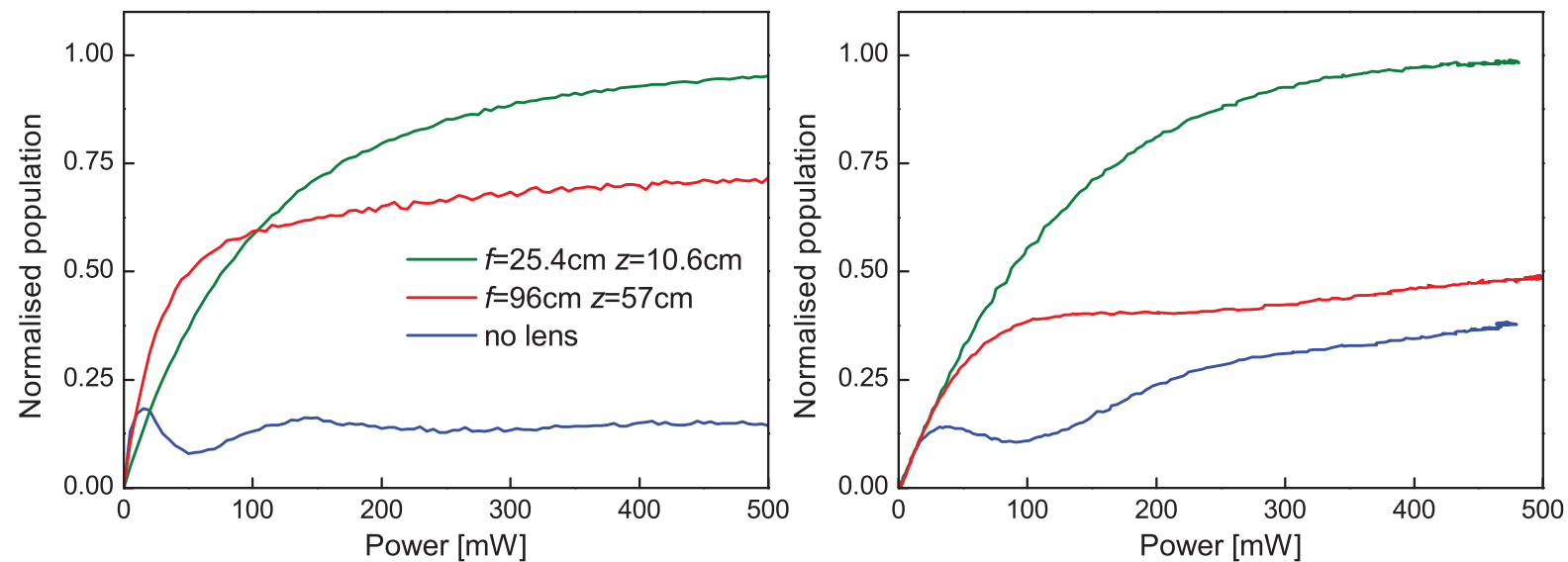

FIG. 8. A comparison of simulated (left) and experimental (right) laser power dependence of the excitation probability for $f=25.4 \mathrm{~cm}, z=10.6 \mathrm{~cm}$ (green); $f=96 \mathrm{~cm}, z=57 \mathrm{~cm}$ (red); and an unfocused laser (blue).

The molecule is therefore promoted to the excited state before undergoing stimulated emission back to the ground state with the result that the population oscillates between the ground and excited state at the Rabi frequency. As the molecular beam moves away from the focal point, the Rabi frequency decreases and the sweep rate increases (as shown in Figures 3 and 4 ) and the probability of the molecule being ex- cited increases. At a distance of $5 \mathrm{~cm}$, the population inversion is complete at sufficiently high laser power.

Using the methods outlined in Sec. III, it is possible to experimentally monitor the level of vibrational excitation in the molecular beam as a function of laser power, where the laser power is varied using a tunable $\lambda / 2$ waveplate in combination with a linear polarizer. The results from such measurements ${ }^{23}$
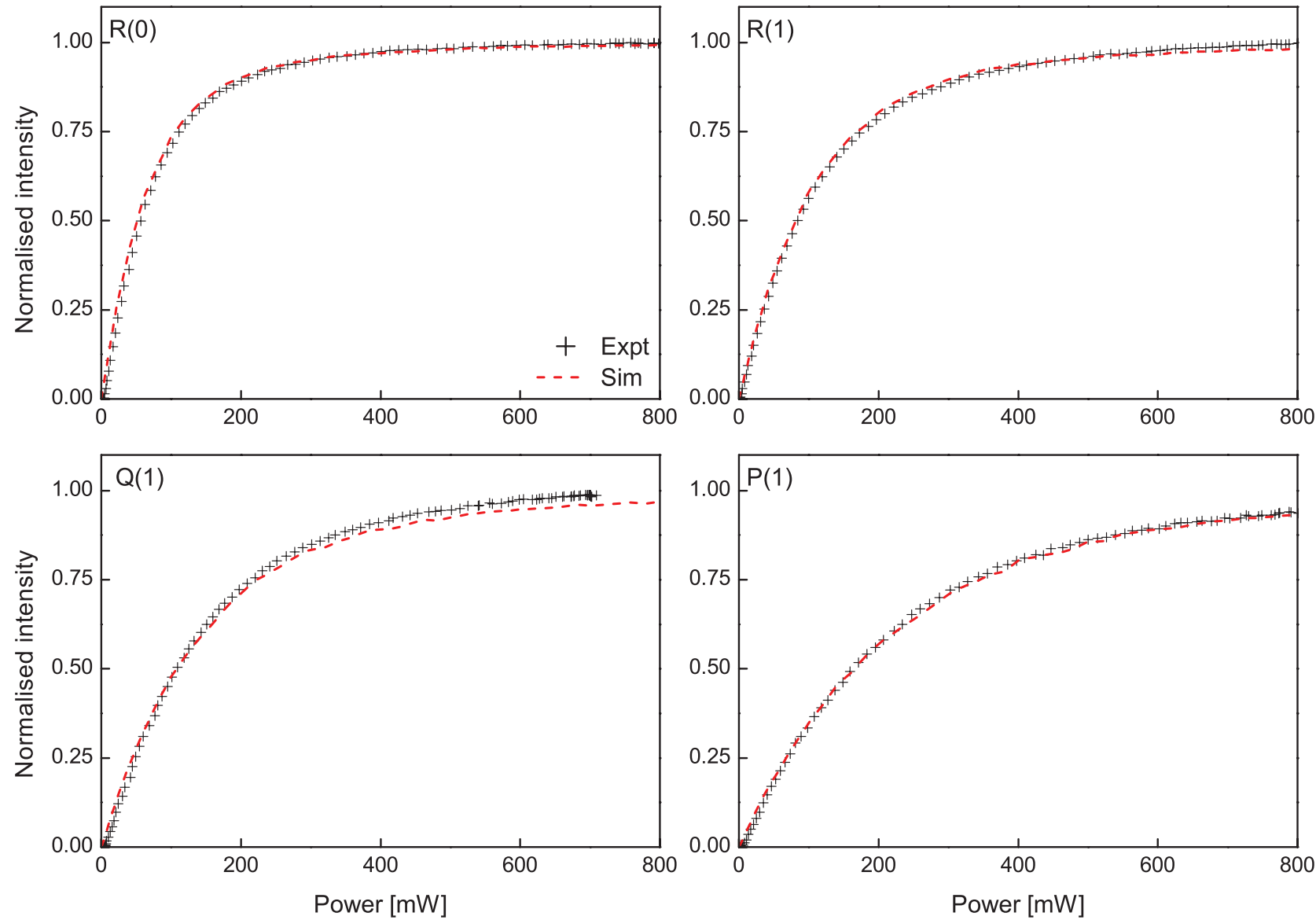

FIG. 9. A comparison of the experimental (black crosses) and calculated (red dashes) laser power dependence of the excitation probability for the R(0) (top left), $\mathrm{R}(1)$ (top right), $\mathrm{Q}(1)$ (bottom left), and $\mathrm{P}(1)$ (bottom right) transitions exciting the $v_{3}$ vibration in $\mathrm{CH}_{4}$. 
for excitation of the $v_{3}$ vibration in $\mathrm{CH}_{4}$ using the $\mathrm{R}(1)$ transition are presented in the right hand panel of Figure 8 for an unfocused laser beam (blue), for a $f=25.4 \mathrm{~cm}$ lens with $z$ $=10.6 \mathrm{~cm}$ (green) and for a $f=96 \mathrm{~cm}$ lens with $z=57 \mathrm{~cm}$ (red). Simulations performed for the same conditions are presented in the left hand panel of Figure 8. Whilst the simulations do not show quantitative agreement with the experimental results, the same qualitative trends are observed. When the laser is unfocused there is evidence for partial Rabi oscillations at low laser powers in both the experimental and simulated fluence curve. In the simulation, the probability reaches a plateau, with less than a quarter of the molecules excited by the laser. The probability is less than 0.5 due to the angular divergence of the molecular beam and the resulting Doppler shift, which causes a significant fraction of the molecular beam to be out of resonance with the excitation laser. These molecules can still be excited experimentally due to power broadening, and therefore the fluence curve is seen to increase, and would reach saturation at higher laser powers.

When the laser is focused with the $f=25.4 \mathrm{~cm}$ lens, the population is completely transferred from the ground state to the excited state through RAP, with the laser exciting the full residual Doppler profile of the molecular beam. Using the $f$ $=96 \mathrm{~cm}$ lens results in more molecules being excited than without focusing, but no population inversion is created. In this case, the total sweep is insufficient for the spread of velocities in the molecular beam, and therefore the conditions for RAP outlined at the end of Sec. II are not satisfied for all molecules.

The measured fluence curves ${ }^{23}$ for the $\mathrm{R}(0)$ (top left), $\mathrm{R}$ (1) (top right), $\mathrm{Q}(1)$ (bottom left), and $\mathrm{P}(1)$ (bottom right) transitions for $v_{3}$ excitation of $\mathrm{CH}_{4}$ are shown as crosses in Figure 9. Each curve reaches saturation when the power is sufficient to create a population inversion, with the different initial gradients of the curve reflecting the different values of the Clebsch-Gordan coefficients in Eq. (11). These experimental curves are compared with those obtained from the simulations (red dashes) in the figure. The agreement between the experimental and simulated curves is excellent. Not only does this comparison offer further confirmation that a population inversion is achieved through the experimental methods used, but the simulations provide a predictive tool for optimizing the experimental conditions as well as providing insight into the requirements to achieve RAP for other transitions and molecules.

\section{SUMMARY}

We present an efficient method for the preparation of intense molecular beams of quantum state selected molecules by IR pumping using RAP. Using $\mathrm{CH}_{4}$ as an example, we demonstrate that RAP can be used to create a population inversion experimentally for both single and double resonance excitation of the $\mathrm{C}-\mathrm{H}$ stretch fundamental $v_{3}$ and the first overtone $2 v_{3}$ with commercially available cw IR OPO sources. A theoretical description of RAP has also been presented for a two level system in terms of the optical Bloch equations. A comparison of the experimental and theoretical results has shown excellent agreement allowing the calculations to be used to optimize the experimental parameters for achieving a population inversion by RAP.

\section{ACKNOWLEDGMENTS}

We gratefully acknowledge financial support provided by the Swiss National Science Foundation (Grant No. 146487/1) and the Ecole Polytechnique Fédérale de Lausanne. We also acknowledge useful discussions with Brad Perkins.

${ }^{1}$ R. D. Beck and A. L. Utz, in Dynamics of Gas-Surface Interactions AtomicLevel Understanding of Scattering Processes at Surfaces, edited by R. Dâiez Muiäno, and H. F. Busnengo (Springer, Berlin, 2013).

${ }^{2}$ L. B. F. Juurlink, D. R. Killelea, and A. L. Utz, Prog. Surf. Sci. 84, 69 (2009).

${ }^{3}$ F. F. Crim, Proc. Natl. Acad. Sci. U.S.A. 105, 12647 (2008).

${ }^{4}$ R. N. Zare, Science 279, 1875 (1998).

${ }^{5}$ L. Chen, H. Ueta, R. Bisson, and R. D. Beck, Faraday Discuss. 157, 285 (2012).

${ }^{6}$ D. R. Killelea, V. L. Campbell, N. S. Shuman, and A. L. Utz, Science 319, 790 (2008).

${ }^{7}$ R. Bisson, M. Sacchi, and R. D. Beck, Phys. Rev. B 82, 121404(R) (2010).

${ }^{8}$ P. Maroni, D. C. Papageorgopoulos, M. Sacchi, T. T. Dang, R. D. Beck, and T. R. Rizzo, Phys. Rev. Lett. 94, 246104 (2005).

${ }^{9}$ B. L. Yoder, R. Bisson, and R. D. Beck, Science 329, 553 (2010).

${ }^{10}$ N. V. Vitanov, T. Halfmann, B. W. Shore, and K. Bergmann, Annu. Rev. Phys. Chem. 52, 763 (2001).

${ }^{11}$ B. W. Shore, K. Bergmann, A. Kuhn, S. Schiemann, J. Oreg, and J. H. Eberly, Phys. Rev. A 45, 5297 (1992).

${ }^{12}$ C. Liedenbaum, S. Stolte, and J. Reuss, Phys. Rep. 178, 1 (1989).

${ }^{13}$ V. S. Malinovsky, and J. L. Krause, Eur. Phys. J. D 14, 147 (2001).

${ }^{14}$ B. W. Shore, M. V. Gromovyy, L. P. Yatsenko, and V. I. Romanenko, Am. J. Phys. 77, 1183 (2009).

${ }^{15}$ A. F. Linskens, N. Dam, B. Sartakov, and J. Reuss, Chem. Phys. Lett. 248, 244 (1996).

${ }^{16}$ A. T. Nguyen, G. D. Chern, D. Budker, and M. Zolotorev, Phys. Rev. A 63, 013406 (2000).

${ }^{17}$ C. R. Ekstrom, C. Kurtsiefer, D. Voigt, O. Dross, T. Pfau, and J. Mlynek, Opt. Commun. 123, 505 (1996).

${ }^{18}$ E. A. McCormack, H. S. Lowth, M. T. Bell, D. Weidmann, and G. A. D. Ritchie, J. Chem. Phys. 137, 034306 (2012).

${ }^{19}$ S. Avrillier, J. M. Raimond, C. J. Bordé, D. Bassi, and G. Scoles, Opt. Commun. 39, 311 (1981).

${ }^{20}$ B. L. Yoder, R. Bisson, P. M. Hundt, and R. D. Beck, J. Chem. Phys. 135, 224703 (2011)

${ }^{21}$ L. Chen, H. Ueta, R. Bisson, and R. D. Beck, Rev. Sci. Instrum. 84, 053902 (2013).

${ }^{22}$ P. M. Hundt, R. Bisson, and R. D. Beck, J. Chem. Phys. 137, 074701 (2012).

${ }^{23}$ B. L. Yoder, Steric Effects in the Chemisorption of Vibrationally Excited Methane on Nickel (Springer, Berlin, 2012).

${ }^{24}$ W. Demtröder, Laser Spectroscopy: Basic Concepts and Instrumentation (Springer, Berlin, 1998).

${ }^{25}$ J. P. C. Kroon, H. A. J. Senhorst, H. C. W. Beijerinck, B. J. Verhaar, and N. F. Verster, Phys. Rev. A 31, 3724 (1985).

${ }^{26}$ R. N. Zare, Angular Momentum Understanding Spatial Aspects in Chemistry and Physics (Wiley, New York, 1988).

${ }^{27}$ L. S. Rothman, I. E. Gordon, A. Barbe, D. C. Benner, P. F. Bernath, M. Birk, V. Boudon, L. R. Brown, A. Campargue, J. P. Champion, K. Chance, L. H. Coudert, V. Dana, V. M. Devi, S. Fally, J. M. Flaud, R. R. Gamache, A. Goldman, D. Jacquemart, I. Kleiner, N. Lacome, W. J. Lafferty, J. Y. Mandin, S. T. Massie, S. N. Mikhailenko, C. E. Miller, N. MoazzenAhmadi, O. V. Naumenko, A. V. Nikitin, J. Orphal, V. I. Perevalov, A. Perrin, A. Predoi-Cross, C. P. Rinsland, M. Rotger, M. Šimečková, M. A. H. Smith, K. Sung, S. A. Tashkun, J. Tennyson, R. A. Toth, A. C. Vandaele, and J. Vander Auwera, J. Quant. Spectrosc. Radiat. Transfer 110, 533 (2009).

${ }^{28}$ M. P. Schmid, P. Maroni, R. D. Beck, and T. R. Rizzo, Rev. Sci. Instrum. 74, 4110 (2003).

${ }^{29}$ A. De Martino, R. Frey, and F. Pradere, Chem. Phys. Lett. 95, 200 (1983). 
${ }^{30}$ C. Boursier, J. Menard, A. Marquette, and F. Menard-Bourcin, J. Mol. Spectrosc. 237, 104 (2006).

${ }^{31}$ A. De Martino, R. Frey, and F. Pradere, Chem. Phys. Lett. 100, 329 (1983).

${ }^{32}$ U. Gaubatz, P. Rudecki, M. Becker, S. Schiemann, M. Külz, and K. Bergmann, Chem. Phys. Lett. 149, 463 (1988).
${ }^{33}$ K. Bergmann, H. Theuer, and B. W. Shore, Rev. Mod. Phys. 70, 1003 (1998).

${ }^{34}$ U. Hübner and G. Kramer, Phys. Rev. A 35, 733 (1987).

${ }^{35}$ P. L. Knight and P. W. Milonni, Phys. Rep. 66, 21 (1980).

${ }^{36}$ J. M. Supplee, Am. J. Phys. 68, 180 (2000).

${ }^{37}$ J. R. Dormand and P. J. Prince, J. Comput. Appl. Math. 6, 19 (1980). 